\title{
EXCESS AND CONJUGATION IN THE STEENROD ALGEBRA
}

\author{
ANDREW M. GALLANT
}

\begin{abstract}
Some formulas concerning conjugation are generalized to certain sub-Hopf-algebras of the Steenrod algebra. A number-theoretic result enables explicit computation of the excess for a certain family of Milnor basis elements. Finally, a family of self-conjugate elements is produced.
\end{abstract}

1. Results. In the mod $p$ Steenrod algebra [4], for $R=\left(r_{1}, \ldots\right)$, let $P_{t}(R)$ be the element in the Milnor basis corresponding to the sequence $\left(0, \ldots, r_{1}, 0, \ldots, r_{2}, 0, \ldots\right)$ whose components are zero except possibly for the elements $r_{i}$ in the it th place. $P_{1}(R)=P(R)$ is the usual Milnor basis element corresponding to the sequence $R$. Let $P_{t}(r)$ be the element whose sequence is $(0, \ldots, r, 0, \ldots)$ where the only nonzero entry is $r$ in the $t$ th place, and let $P_{t}^{s}=P_{t}\left(p^{s}\right)$. The dimension of $P_{t}(R)$ is given by $2 \sum r_{i}\left(p^{i t}-1\right)$ and the excess by $2 \sum r_{i}$ for $p$ odd, with the usual modifications being needed for $p=2$, and sequences will be ordered lexicographically from the right. It will be convenient to generalize Davis' notation [2] to $S_{t}(n)$ for the sum of all elements $P_{t}(R)$ in dimension $n$. Also, let $A_{t}$ denote the set of all $P_{t}(R)$ for a fixed $t$. Adams and Margolis show that $A_{t}$ is a sub-Hopf-algebra of the Steenrod algebra [1]. This may also be seen directly by observing that in both the Cartan formula and the Milnor multiplication matrix, the only possibly nonzero elements must have indices which are multiples of $t$.

Milnor [4] defines a canonical antiautomorphism $\chi$ on the Steenrod algebra and shows that $\chi\left(P_{1}(r)\right)=(-1)^{r} S_{1}(n)$, where $n=\operatorname{dim}\left(P_{1}(r)\right)$. This result can be generalized to:

Proposition 1. $\chi\left(P_{t}(r)\right)=(-1)^{r} S_{t}(n)$, where $n=\operatorname{dim}\left(P_{t}(r)\right)$.

It will be shown below that $\chi$ is in fact an antiautomorphism of $A_{t}$. It can also be shown that Davis' formulas [2] can be generalized. The proofs are virtually identical to his and so will not be given here.

Corollary 1.

$$
P_{t}(m) S_{t}(n)=\sum\left(\begin{array}{c}
\sum p^{i t} r_{i} \\
p^{t} m
\end{array}\right) P_{t}(R)
$$

where the sum is over all $R$ of the correct dimension.

Received by the editors August 14, 1978 and, in revised form, October 11, 1978.

AMS (MOS) subject classifications (1970). Primary 55G10; Secondary $05 A 10$.

Key words and phrases. Steenrod algebra, excess, conjugation, Milnor basis. 
(b)

$$
\chi\left(P_{t}\left(1+p^{t}+\cdots+p^{(n-1) t}\right)\right)=(-1)^{n} P_{t}\left(p^{(n-1) t}\right) \cdots P_{t}(1) .
$$

Kraines [3] defines the notion of excess for operations expressed in the Milnor basis. The principal result states that the excess (in the usual sense) of a linear combination of such elements is the minimum of the excesses (as defined above) of the terms. For $\chi\left(P_{1}(r)\right)$, another result states that the excess is that of a solution of $(p-1) r=\Sigma s_{i}\left(p^{i}-1\right)$ which minimizes $\Sigma s_{i}[3$, Corollary 5]. In the next section we study a more general situation, and the results may be summarized as follows:

Proposition 2. For each $r$ and fixed $b$, there is exactly one solution $A=\left(a_{1}, a_{2}, \ldots\right)$ of the equation in nonnegative integers

$$
(b-1) r=\sum a_{k}\left(b^{k}-1\right)
$$

which has the form

$$
a_{k}=0 \text { for } k<j, \quad 1 \leqslant a_{j} \leqslant b, \text { and } 0 \leqslant a_{k} \leqslant b-1 \text { for } k>j, \text { for some } j \text {, }
$$

for which $A$ is simultaneously maximal in order and minimal in excess.

Corollary 2. $\operatorname{ex}\left(\chi\left(P_{t}(r)\right)\right)=\operatorname{ex}\left(P_{t}(A)\right)$, where $A$ has form (1.2) for $b=p^{t}$ in (1.1).

This solution can be easily computed by the division algorithm for any choice of $r$ and $b$, and a particularly nice result occurs for $P_{t}^{s}$.

Corollary 3. $\operatorname{ex}\left(\chi\left(P_{t}^{s}\right)\right)=\min \left(\operatorname{ex}\left(P_{t}^{s}\right), \operatorname{ex}\left(P_{t}^{t}\right)\right)$.

In [4], Milnor gives a procedure for finding the conjugate of an arbitrary element $P(R)$ as a linear combination of terms $P(S(Y))$. We will show the following:

THEOREM. For each term $P_{t}(S)$ in $\chi\left(P_{t}(R)\right)$, then, for $S \neq R, \operatorname{ex}\left(P_{t}(S)\right)<$ $\operatorname{ex}\left(P_{t}(R)\right)$ and $P_{t}(S)>P_{t}(R)$.

Applying this result to the solutions $A$ of minimal excess and maximal order produces a family of self-conjugate elements.

Corollary 4. For each $r, p$, and $t$, let $A$ be the solution of form (1.2) of (1.1), with $b=p^{t}$. Then $\chi\left(P_{t}(A)\right)=(-1)^{\Sigma a_{i}} P_{t}(A)$.

2. Number theory. We will want to examine all solutions of equation (1.1). Define the excess of a solution as the sum of its elements, and order all solutions for $r$ and $b$ fixed lexicographically from the right. For example, if $r=7$ and $b=2$, the solutions are ordered $(7)<(4,1)<(1,2)<(0,0,1)$. It is convenient to rewrite (1.1) as

$$
r=\sum a_{k} b_{k}, \quad \text { where } b_{k}=\left(b^{k}-1\right) /(b-1)=1+b+\cdots+b^{k-1} \text {. }
$$


The following elementary identities are easily established:

$$
\begin{gathered}
b b_{k}=b_{k+1}-1, \\
a_{j} b_{j}+b b_{k}=b b_{j-1}+\left(a_{j}-1\right) b_{j}+b_{k+1}, \quad \text { for } k \geqslant j \geqslant 2, \quad a_{j} \geqslant 1, \\
p^{k t+n}=1+\left(p^{t}-p^{n}\right)\left(p^{t}\right)_{k}+\left(p^{n}-1\right)\left(p^{t}\right)_{k+1}, \\
\quad \text { for } 0 \leqslant n<t, \quad k \geqslant 1 .
\end{gathered}
$$

The solutions of (2.1) are totally ordered and finite in number, so there is a maximal solution, and it may be constructed by an iterated division algorithm: define $a_{k}$ as $k$ decreases by $c_{k+1}=a_{k} b_{k}+c_{k}$, where $0<c_{k}<b_{k}$; let the process commence when $k$ is such that $b_{k+1}>r \geqslant b_{k}$, letting $c_{k+1}=r$; and note that the process terminates since $c_{k+1} \geqslant b_{k}>c_{k}$, so that the $c_{k}$ decrease as $k$ decreases. It is clear by construction that this solution is maximal, and it is apparent from (2.2) that for each $k, a_{k}<b$, and that if $a_{j}=b$ for some $j$, then $a_{k}=0$ for $k<j$, because there is no remainder. This provides a way of recognizing maximal solutions by their "digits", namely the form (1.2).

It also turns out that for $r$ and $b$ fixed there is only one solution of form (1.2), and to prove this uniqueness it suffices to show that $\sum_{i=j}^{k} a_{i} b_{i}<b_{k+1}$, for $A$ of form (1.2). Indeed, by repeated application of (2.2), it follows that

$$
\sum_{i=j}^{k} a_{i} b_{i} \leqslant b b_{j}+\sum_{i=j+1}^{k}(b-1) b_{i}=b_{k+1}-(k-j+1)<b_{k+1} .
$$

An important property of this solution is that it has minimal excess, and this may be seen by transforming a nonmaximal solution into another solution of higher order but no higher excess. Specifically, a nonmaximal solution must have a "digit" $a_{k} \geqslant b$, and if $a_{k}=b$, then for some $j<k$ there is an $a_{j} \geqslant 1$. Applying (2.3), with $j=k$ if $a_{k} \geqslant b+1$, yields a new solution of higher order and equal excess, unless $A=\left(a_{1}, a_{2}, \ldots\right)$ is such that $\left(0, a_{2}, a_{3}, \ldots\right)$ has form (1.2). In that case, applying (2.2) produces a new solution of higher order and lower excess, if $A$ is not already of form (1.2). Because there are only finitely many solutions, repeated application of these transformations must produce the maximal solution. In fact, all solutions may be generated by (2.2), (2.3), and their inverses.

At this point, the warning is needed that although excess and order seem to be inversely related, they do not determine each other. Two examples are the solutions $(0, b+1)<(b, 0,1)$ and $(0, b+2)<(2 b+1,0,1)$, for $r=$ $(b+1)^{2}$ and $r=(b+1)(b+2)$, respectively. The first example shows two solutions of minimal excess, and in the second, the solution of lower excess is also of lower order. These are the lowest dimensional examples of their types.

The above demonstrations provide the proof of Proposition 2.

Two specific results needed for later come from maximality, (2.4), and letting $s=k t+n$. 
LEMMA 1. (a) For $r \leqslant b$, the unique solution is $(r, 0, \ldots)$.

(b) For $r=p^{s}$ and $b=p^{t}$, the maximal solution has excess $p^{t}$ when $s>t$.

3. Conjugation. Consider Milnor's equations [4]

$$
r_{i}=\sum_{n} \sum_{\alpha} y_{\alpha} \sum_{j=1}^{l(\alpha)} \delta_{i, \alpha(j)} p^{\sigma(j)}
$$

where $\alpha=(\alpha(1), \ldots, \alpha(l(\alpha)))$ is an ordered partition of $n, \sigma(j)=\sum_{i=1}^{j-1} \alpha(i)$, and each $y_{\alpha}$ is a nonnegative integer. Let $S(Y)=\left(s_{1}, \ldots\right)$ where $s_{n}=\Sigma_{\alpha} y_{\alpha}$, and let $b(Y)=\Pi_{n} s_{n} ! / \Pi_{n} \Pi_{\alpha} y_{\alpha}$ !. Then Milnor's result on conjugation [2, Theorem 5] states that

$$
\chi\left(P_{1}(R)\right)=(-1)^{\Sigma r_{i}} \sum b(Y) P_{1}(S(Y)),
$$

where the summation ranges over all solutions $Y=\left\{y_{\alpha}\right\}$ to the system (*). Milnor observes that for $r_{i}$, the coefficient of $y_{\alpha}$ is positive if and only if $i$ is in $\alpha$. It follows that if $r_{i}=0$, then $y_{\alpha}=0$ for all $\alpha$ containing an $i$.

LEMMA 2. $\chi$ is an antiautomorphism on $A_{t}$.

Proof. For an element $P_{1}(R)$ of $A_{t}, r_{i}=0$ unless $i=k t$. Then $y_{\alpha} \neq 0$ only for partitions $\alpha$ all of whose elements are multiples of $t$, which makes $\alpha$ a partition of a multiple of $t$, and therefore $s_{i}=0$ unless $i=k t$.

Proof of Proposition 1. Again using (*), it is easy to see that $y_{\alpha}=0$ unless $\alpha$ has the form $(t),(t, t)$, etc., whence $s_{t}=y_{t}, s_{2 t}=y_{t, t}$, etc., $b(Y)=1$, and $(*)$ reduces to a single equation

$$
r_{t}=\sum s_{k t}\left(1+p^{t}+\cdots+p^{(k-1) t}\right) \text {. }
$$

However, this equation is just of form (2.1). For clarity, in form (1.1) it becomes

$$
\left(p^{t}-1\right) r=\sum s_{k t}\left(p^{k t}-1\right)
$$

which is just the restriction on dimension. This is Milnor's result [4, Corollary 6] for $t=1$, and the proof follows his.

Proof OF TheOREM. By Lemma 1 it suffices to prove the result for elements $P(R)=P_{1}(R)$. Note that if only the partitions $\alpha=(n)$ are used, then (*) implies that $r_{n}=y_{n}=s_{n}, b(Y)=1$, and $P(R)$ is a term in $\chi(P(R))$ with coefficient $(-1)^{\Sigma r_{i}}$. To prove the result for excess add the equations (*) for all $i$. Since $\sum_{i} \delta_{i, \alpha(j)}=1$ and $\sum_{j=1}^{l(\alpha)} p^{\sigma(j)} \geqslant 1$, then

$$
\sum r_{i}=\sum_{n} \sum_{\alpha} y_{\alpha} \sum_{j=1}^{l(\alpha)} p^{\sigma(j)} \sum_{i} \delta_{i, \alpha(j)} \geqslant \sum_{n} \sum_{\alpha} y_{\alpha}=\sum_{n} s_{n} .
$$

Equality only occurs when all available partitions have length one, which gives the term $P(R)$. A partition of length greater than one gives $p^{\sigma(j)}>1$, thus lowering excess. For order, assume for $n \geqslant k$ that $r_{n}=y_{n}=s_{n}$ and that 
$y_{\alpha}=0$ if $\alpha$ is a partition of $n$ other than $(n)$. For a fixed $Y$ and $S$, this is true for some $k$, since $r_{n}=y_{n}=s_{n}=0$ for $n$ large enough, for instance, if $p^{n}-1>\sum r_{i}\left(p^{i}-1\right)$. This being the case, then either the above is also true for $n>k-1$, or else $r_{k-1}=y_{k-1}<s_{k-1}$. Assuming this, then the former being true would produce $R=S(Y)$; whereas should the latter occur for some $k-1$, then $R<S(Y)$. But examining (*) for $r_{k-1}$ shows that $r_{k-1}=$ $y_{k-1}$, since the only partition with a $k-1$ and $y_{\alpha} \neq 0$ must be $\alpha=(k-1)$. Then, either $y_{\alpha}=0$ for all other partitions of $k-1$, or else for some such partition $y_{\alpha} \neq 0$, implying that $s_{k-1}>y_{k-1}=r_{k-1}$.

4. Excess. Using Kraines' results on excess [3], we see from Proposition 1 that the excess of $\chi\left(P_{t}(r)\right)$ is that of the term of lowest excess in $S_{t}(n)$. This corresponds to finding the solution of minimal excess of (1.1) with $b=p^{t}$, that equation being the dimensional restriction. Proposition 2 gives the required solution in explicit form, thus proving Corollary 2. Corollary 3 is proved by appealing to Lemma 1 , and Corollary 4 is a consequence of the theorem and the fact that $P_{t}(A)$ is maximal in its dimension in $A_{t}$.

5. Order. There are two usual lexicographic orders, right and left. Specifically, $P(R)>_{R} P(S)\left(P(R)>_{L} P(S)\right)$ if there is some $k$ such that $r_{k}>s_{k}$ and for all $j>k(j<k)$ it is true that $r_{j}=s_{j}$. For instance, if $P(T)$ is a term in $P(R) \cdot P(S)$, then it is easy to see from the Milnor multiplication matrix that, if coefficients are neglected, we have $P(T) \geqslant_{R} P(R+S)>_{L} P(T)$. The theorem may then be restated:

Restatement of TheOREM. For each term $P_{t}(S)$ in $\chi\left(P_{t}(R)\right)$, then for $S \neq R, \operatorname{ex}\left(P_{t}(S)\right)<\operatorname{ex}\left(P_{t}(R)\right)$ and $P_{t}(S)>_{R} P_{t}(R)>_{L} P_{t}(S)$.

Proof. Clearly $s_{1}=y_{1} \leqslant r_{1}$. Assume for $n \leqslant k$ that $s_{n}=y_{n}=r_{n}$, and that $y_{\alpha}=0$ if $\alpha$ is any partition containing $n$ other than (n). Therefore, $y_{\alpha}=0$ if $\alpha$ is any partition of $k+1$ other than $(k+1)$, since it contains such an $n$. This implies that $s_{k+1}=y_{k+1} \leqslant r_{k+1}$. By (*), equality establishes the inductive step for $n=k+1$, while inequality proves the order.

For fixed $r$ and $b$, it is clear that $(r, 0,0, \ldots)$ is right-minimal and left-maximal, and that it carries maximal excess. It has been shown that right-maximal elements have minimal excess, are self-conjugate, and have an explicit presentation. However, left-minimal elements, although self-conjugate, are in general different from right-maximal elements and so do not carry minimal excess. Consider the two sequences $(1,0,0,1)>_{L}\left(0, b^{2}-b+\right.$ $1,1)$, for $r=b^{3}+b^{2}+b+2$, as an example. The former is the unique element of minimal excess, which may be verified by inspection, but it is not left-minimal. For $b=2(r=16)$, this is the lowest dimensional example of its type. 


\section{REFERENCES}

1. J. F. Adams and H. R. Margolis, Sub-Hopf-algebras of the Steenrod algebra, Proc. Cambridge Philos. Soc. 76 (1974), 45-52. MR 49 \#6238.

2. D. M. Davis, The antiautomorphism of the Steenrod algebra, Proc. Amer. Math. Soc. 44 (1974), 235-236. MR 48 \# 7276.

3. D. Kraines, On excess in the Milnor basis, Bull. London Math. Soc. 3 (1971), 363-365. MR 45 \#9317.

4. J. Milnor, The Steenrod algebra and its dual, Ann. of Math. (2) 67 (1958), 150-171. MR 20 \#6092.

Department of Mathematics, College of St. Francis, Joliet, IllnNois 60435 